\title{
Existence and uniqueness results for Liénards equation having a dead band
}

\author{
Sandqvist, Allan; Andersen, Kurt Munk
}

Published in:

IEEE Transactions on Circuits and Systems

Link to article, DOI:

10.1109/TCS.1980.1084775

Publication date:

1980

Document Version

Publisher's PDF, also known as Version of record

Link back to DTU Orbit

Citation (APA):

Sandqvist, A., \& Andersen, K. M. (1980). Existence and uniqueness results for Liénards equation having a dead band. IEEE Transactions on Circuits and Systems, 27(12), 1251-1254.

https://doi.org/10.1109/TCS.1980.1084775

\section{General rights}

Copyright and moral rights for the publications made accessible in the public portal are retained by the authors and/or other copyright owners and it is a condition of accessing publications that users recognise and abide by the legal requirements associated with these rights.

- Users may download and print one copy of any publication from the public portal for the purpose of private study or research.

- You may not further distribute the material or use it for any profit-making activity or commercial gain

- You may freely distribute the URL identifying the publication in the public portal

If you believe that this document breaches copyright please contact us providing details, and we will remove access to the work immediately and investigate your claim 


\title{
Existence and Uniqueness Results for Liénard's Equation Having a Dead Band
}

\author{
ALLAN SANDQVIST AND KURT MUNK ANDERSEN
}

\begin{abstract}
In the first part of the present paper we consider general systems of first-order autonomous differential equations and generalize a uniqueness criterion by Dulac concerning periodic solutions to equations of the form $\dot{x}=P(x, y), \dot{y}=Q(x, y)$. In the second part we use this result to generalize a uniqueness theorem by de Figueiredo concerning periodic solutions to Liénard's equation $\ddot{x}+f(x) \dot{x}+g(x)=0$. By our method we are able to avoid the hitherto usual condition $x g(x)>0, x \neq 0$, which excludes the possibility for the equation to have a dead band. Finally, we prove an existence theorem concerning periodic solutions to such equations. The use of the theorems is illustrated by a simple example in the last section.
\end{abstract}

\section{INTRODUCTION}

In [1] the author proves a theorem concerning the existence and uniqueness of periodic solutions to Liénard's equation

$$
\ddot{x}+f(x) \dot{x}+g(x)=0 .
$$

It is an essential condition for the method in [1] to work that $x g(x)>0, x \neq 0$, which unfortunately excludes the situation-important from a technical viewpoint-in which $g(x) \equiv 0$ in some interval around 0 , i.e., the equation has a dead band. The main aim of the present paper is to prove a uniqueness theorem analogous to the one contained in [1], applying a different method which in particular allows the equation to have a dead band. In addition we prove a simple existence theorem, using partly the same idea which is used in deriving the uniqueness conditions. Our method is based upon some resultscontained in the following section-concerning general systems of first-order differential equations. These results are obtained by appropriately combining some well-known ideas of Lyapunov and Dulac.

\section{SOME EXTENSIONS OF THE UNIQUENESS THEOREM OF DULAC}

We consider an autonomous system

$$
\underline{\dot{x}}=\underline{f}(\underline{x}), \quad \underline{x} \in D
$$

where $f: D \rightarrow R^{k}$ in some open domain $D \subseteq R^{k}$ and assume that $f$ is continuous with continuous partial derivatives $f_{i, x_{i}}^{\prime}$ in $\bar{D}$. Note that $f$ is not assumed to be continuously differentiable.

Let $V: D \rightarrow R$ be any function which is continuous in $D$ together with its partial derivatives of first order.

Manuscript received November 1, 1978; revised May 27, 1980.

The authors are with the Mathematical Institute, The Technical University of Denmark, DK-2800 Lyngby, Denmark.
Definition: A component of the open set

$$
\{\underline{x} \in D \mid V(\underline{x})>0\}
$$

is called a $p$-cell (with respect to $V$ ).

Lemma: Let $\Omega \subseteq D$ be a $p$-cell. If there exists a continuous function $H: D \rightarrow R$ such that the trajectory derivative $V^{*} \triangleq f \cdot \underline{\operatorname{grad}} V$ satisfies the inequality

$$
V^{*} \geqslant H V
$$

at every point of $\Omega$, then $\Omega$ is positively invariant.

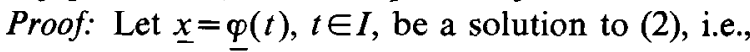

$$
\dot{\varphi}_{i}(t)=f_{i}(\underline{\varphi}(t)), \quad t \in I, \quad i=1, \cdots, k .
$$

Let $\underline{x}_{0}=\underline{\varphi}\left(t_{0}\right) \in \Omega$ for some $t_{0} \in I$. Introducing the function

$$
\tilde{V}(\underline{x})=V^{*}(\underline{x})-H(\underline{x}) V(\underline{x}), \quad \underline{x} \in D
$$

we define

By (4)

$$
\begin{aligned}
& v(t)=V(\underline{\varphi}(t)) \\
& \tilde{v}(t)=\tilde{V}(\underline{\varphi}(t)) \\
& h(t)=H(\underline{\varphi}(t)), \quad t \in I .
\end{aligned}
$$

$$
\frac{d v}{d t}-h(t) v(t)=\tilde{v}(t), \quad t \in I
$$

from which it follows that

$$
v(t)=e^{\int_{t_{0}}^{t} h(\tau) d \tau}\left\{\int_{t_{0}}^{t} e^{-\int_{t_{0}}^{\tau} h(s) d s} \tilde{v}(\tau) d \tau+v\left(t_{0}\right)\right\}, \quad t \in I .
$$

Since $v\left(t_{0}\right)>0$ and $\tilde{v}(t) \geqslant 0$ as long as $\varphi(t) \in \Omega$, (5) implies that $v(t)>0$ for all $t \in I \cap\left[t_{0},+\infty[\right.$, i.e., $\varphi(t) \in \Omega$ for all $t \in I \cap\left[t_{0},+\infty[\right.$.

Theorem 1: A p-cell with respect to $V$ in which the inequality

$$
\operatorname{div}\left(V^{-1} f\right) \leqslant 0
$$

holds, is positively invariant.

Proof: Since

$$
\begin{aligned}
\operatorname{div}\left(V^{-1} \underline{f}\right) & =V^{-2}\{V \operatorname{div} \underline{f}-\underline{f} \cdot \underline{\operatorname{grad}} V\} \\
& =V^{-2}\left\{V \operatorname{div} \underline{f}-V^{*}\right\} \leqslant 0
\end{aligned}
$$

we have

$$
V^{*} \geqslant(\operatorname{div} f) V
$$

in the $p$-cell, i.e., the theorem is a corollary to the lemma. 
From now on we consider only the case $k=2$, i.e., we consider

$$
\left.\begin{array}{l}
\dot{x}=P(x, y) \\
\dot{y}=Q(x, y)
\end{array}\right\}, \quad(x, y) \in D
$$

where $D$ is some open domain in $R^{2}$.

As before we assume, that $P, Q, P_{x}$, and $Q_{y}$ are continuous in $D$ and in addition we assume that $P$ and $Q$ satisfy a condition (e.g., a Lipschitz condition) which assures local uniqueness of the solutions of (7) in $D$.

The following theorem is a modification of the wellknown uniqueness theorem of Dulac [2].

Theorem 2: Let $\Omega \subseteq D$ be a $p$-cell with respect to some function $V$ which is continuously differentiable in $D$. If there are no critical points for the system (7) in $\Omega$ and if the inequality

$$
\operatorname{div}\left(V^{-1} P, V^{-1} Q\right) \leqslant 0
$$

holds everywhere in $\Omega$, then

i) if $\Gamma$ is a closed trajectory for the system (7) containing a point of $\Omega$, then $\Gamma \subset \Omega$;

ii) if $\Gamma_{1}$ and $\Gamma_{2}$ are closed trajectories in $\Omega$ with $\Gamma_{1}$ lying in the interior of $\Gamma_{2}$ such that the open annular domain $\omega$ with boundary $\Gamma_{1} \cup \Gamma_{2}$ is contained in $D$ and contains no critical points then

(iia) $\omega \subset \Omega$,

(iib) the equality-sign in (8) holds everywhere in $\bar{\omega}$,

(iic) $\bar{\omega}$ is a union of closed trajectories.

Proof: i) follows directly from Theorem 1. If $p$ is a point in $\omega$, the negative limitset $L\left(\Gamma^{-}\right)$for the trajectory $\Gamma$ through $p$ is a closed trajectory contained in $\bar{\omega}$. Since $\Omega$ is connected, two arbitrary points $p_{1}$ on $\Gamma_{1}$ and $p_{2}$ on $\Gamma_{2}$ can be joined by some continuous curve $k$ lying in $\Omega$-and we may assume that $k \subset \Omega \cap \bar{\omega}$. Moreover, $\bar{\omega}$ contains no critical points. Hence $L\left(\Gamma^{-}\right)$must intersect $k$. Either $L\left(\Gamma^{-}\right)=$ $\Gamma$ or $L\left(\Gamma^{-}\right)$is a limit-cycle. In either case $\Gamma^{-}$must intersect $k$ and, therefore, contain a point of $\Omega$. From Theorem 1 it follows that $p \in \Omega$, proving (iia).

By the theorem of Gauss we get

$$
\int_{\bar{\omega}} \operatorname{div}\left(V^{-1} P, V^{-1} Q\right) d \sigma=\int_{\partial \bar{\omega}}-V^{-1} Q d x+V^{-1} P d y=0 .
$$

Since the function $\operatorname{div}\left(V^{-1} P, V^{-1} Q\right)$ is continuous and nonpositive in $\bar{\omega}$ it vanishes everywhere in $\bar{\omega}$, proving (iib).

Let $\left(x_{0}, y_{0}\right)$ be an arbitrary point of $\bar{\omega}$. The function

$$
u(x, y)=\int_{\left(x_{0}, y_{0}\right)}^{(x, y)} V^{-1}\{-Q(x, y) d x+P(x, y) d y\},
$$

$$
(x, y) \in \bar{\omega}
$$

where the line integral is evaluated along some piecewise regular $C^{1}$-curve in $\bar{\omega}$, is continuous in $\bar{\omega}$ and a primitive to $V^{-1}(-Q d x+P d y)$ in $\omega$, since the value of the integral does not depend on the path connecting $\left(x_{0}, y_{0}\right)$ and $(x, y)$. The last assertion follows from (iib) and the fact that $\Gamma_{1}$ is a closed trajectory. If a point $p$ of $\omega$ did not belong to a closed trajectory, then the positive and the negative limit sets for the trajectory through $p$ would be two adjacent closed trajectories in $\bar{\omega}$ and $u(\bar{x}, y)$ would have the same value on both, which contradicts the fact that $\operatorname{grad} u=V^{-1}(-Q, P) \neq \underline{0}$ in $\omega$. This proves (iic).

Now assume that $P$ and $\bar{Q}$ are both continuously differentiable in $D$ and $\Gamma \subset D$ is a closed trajectory corresponding to the periodic solution

$$
(x, y)=(\varphi(t), \psi(t)), \quad t \in R
$$

with period $T>0$. Letting $H=\operatorname{div}(P, Q)$ and

$$
\begin{aligned}
h(t) & \triangleq H(\varphi(t), \psi(t)) \\
& =\operatorname{div}(P, Q) \circ(\varphi(t), \psi(t)), \quad t \in R
\end{aligned}
$$

we get by (5) $($ since $v(T)=v(0))$

$$
v(0)\left[e^{-\mu T}-1\right]=\int_{0}^{T} e^{-\int_{0}^{t} h(\tau) d \tau} \tilde{v}(t) d t
$$

where

$$
\mu=\frac{1}{T} \int_{0}^{T} h(t) d t+\frac{1}{T} \int_{0}^{T} \operatorname{div}(P, Q) \circ(\varphi(t), \psi(t)) d t
$$

is the characteristic exponent for $\Gamma$, and

$$
\tilde{v}(t)=[(P, Q) \cdot \underline{\operatorname{grad}} V-V \operatorname{div}(P, Q)] \circ(\varphi(t), \psi(t)),
$$

We are now in a position to prove a theorem which is a straightforward extension of the uniqueness theorem of Dulac:

Theorem 3: If

i) $D_{1} \subseteq D$ is an annular domain which contains no critical points for the system (7).

ii) $V$ is nonnegative in $D_{1}$.

iii) The integral

$$
\int_{0}^{T} e^{-S_{0}^{t} h(\tau) d \tau} \tilde{v}(t) d t
$$

is strictly positive along any closed trajectory in $D_{1}$ (where $T$ is the period of the corresponding periodic solution and $h$ and $\tilde{v}$ are given by (9) and (11), respectively) then $D_{1}$ contains at most one (complete) closed trajectory, and this trajectory is positively asymptotically stable.

Proof: The last assertion follows from (10), which implies $\mu<0$. Now assume that there exist at least two different closed trajectories $\Gamma_{1}$ and $\Gamma_{2}$ in $D_{1}$, with $\Gamma_{1}$ in the interior of $\Gamma_{2}$, and let $\omega \subset D_{1}$ be the annular domain with boundary $\Gamma_{1} \cup \Gamma_{2}$. By the above remark both $\Gamma_{1}$ and $\Gamma_{2}$ are positively asymptotically stable, hence $\omega$ cannot be a union of closed trajectories. Hence it is possible to find a point $p$ in $\omega$ such that the (maximal) trajectory $\Gamma$ through $p$ is not closed. It follows that the limit sets $L\left(\Gamma^{+}\right)$and $\bar{L}\left(\Gamma^{-}\right)$are adjacent closed trajectories which both are positively asymptotically stable. It is well known however, that this situation cannot occur (see, e.g., [3]).

\section{IIII. Some Results for the Equation of LiÉnARD}

By means of Theorem 2 in the previous section we get the following uniqueness theorem: 
Theorem 4: Let $f$ and $g$ be continuous functions in an open interval $I \subseteq R$ around 0 and assume further that $f$ and $g$ satisfy a condition (e.g., a Lipschitz condition) assuring local uniqueness of the solutions to the Lienard equation

$$
\ddot{x}+f(x) \dot{x}+g(x)=0 .
$$

Let

$$
F(x)=\int_{0}^{x} f(\xi) d \xi, \quad x \in I
$$

and

$$
G(x)=\int_{0}^{x} g(\xi) d \xi, \quad x \in I .
$$

Let $a_{1}$ and $a_{2}$ be nonnegative real numbers, such that $\left[-a_{1}, a_{2}\right] \subset I$. Equation (12) has at most one nontrivial periodic solution (up to translations in $t$ ) if

(ia) $g(x)=0, \quad x \in\left[-a_{1}, a_{2}\right]$,

(ib) $x g(x)>0, \quad x \in I \backslash\left[-a_{1}, a_{2}\right]$,

(iia) $2 G(x) f(x)-F(x) g(x) \geqslant 0, \quad x \in I$,

(iib) Any neighborhood $]-\alpha_{1}, \alpha_{2}\left[\right.$ of $\left[-a_{1}, a_{2}\right]$ contains a point at which $2 G(x) f(x)-F(x) g(x)>0$.

(Note, that $2 G(x) f(x)-F(x) g(x)=0, x \in\left[-a_{1}, a_{2}\right]$ by

(ia)). The case $\left[-a_{1}, a_{2}\right]=\{0\}$ is not excluded.

Proof: Equation (12) is equivalent to the system

$$
\begin{aligned}
& \dot{x}=P(x, y)=y \\
& \dot{y}=Q(x, y)=-f(x) y-g(x)
\end{aligned}
$$

where $P$ and $Q$ satisfy the conditions of Theorem 2 in the open domain $D=I \times R$.

Let

$$
V(x, y)=y^{2}+y F(x)+2 G(x), \quad(x, y) \in D .
$$

This is a continuously differentiable function and by computation

$$
\begin{aligned}
\tilde{V}(x, y) & =-V^{2} \operatorname{div}\left(V^{-1} P, V^{-1} Q\right) \\
& =(P, Q) \cdot \underline{\operatorname{grad}} V-V \operatorname{div}(P, Q) \\
& =2 G(x) f(x)-F(x) g(x) .
\end{aligned}
$$

Note that $\tilde{V}(x, y)$ is a function of $x$ alone, i.e., $\tilde{V}$ must be positive-in view of (iib)-on straight lines $x=x_{0}$, where $x_{0}$ can be chosen arbitrarily close to $\left[-a_{1}, a_{2}\right]$.

The points on the linesegment $l=\left[-a_{1}, a_{2}\right] \times\{0\}$ form the set of critical points for the system (13). Any closed trajectory must encircle $l$ and hence intersect the positive part of the $y$ axis, where $V>0$. By Theorem 1 the $p$-cell containing the positive part of the $y$ axis must contain every closed trajectory of the system (13). However, by the remark above and the second part of Theorem 2 the possibility of more than one closed trajectory is ruled out.

Remark: For comparison we briefly mention the theorem 2 in [1] on uniqueness: Let $f$ and $g$ be continuous functions on the real line, satisfying a condition (such as a Lipschitz condition) assuring local uniqueness of the solutions to (12). There will be at most one (nontrivial) periodic solution up to translations in time if the following conditions are satisfied. (a)

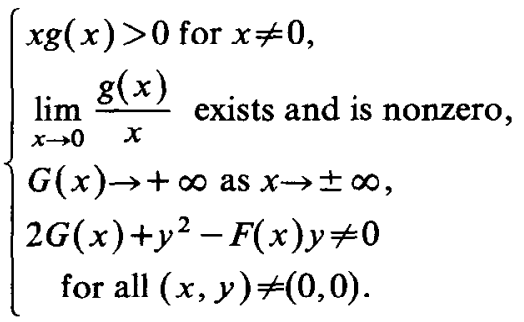

(b) $2 G(x) f(x)-F(x) g(x)>0$ for all real $x$, except possibly on a set of Lebesgue measure zero.

Theorem 4 is a generalization of this result in the case $\left[-a_{1}, a_{2}\right]=\{0\}$. If $\left[-a_{1}, a_{2}\right] \neq\{0\}(12)$ has a dead band and this case cannot be dealt with by means of theorem 2 in [1].

By means of the function $V$ we can prove the following existence theorem, too.

Theorem 5: Let $I=R$, and assume that $f$ and $g$ satisfy the conditions of Theorem 4, possibly with the exception of (iib), and in addition the conditions

(iii) $G(x) \rightarrow+\infty$ for $x \rightarrow \pm \infty$,

(iv) $f(x) \leqslant 0$ in some neighborhood $]-\alpha_{1}, \alpha_{2}[$ of $\left[-a_{1}, a_{2}\right]$,

(v) there exists a positive number a such that

$$
f(x) \geqslant 0, \quad x \in R \backslash[-a, a], \text { and }
$$

$[F(x)]^{2}-8 G(x) \geqslant 0$,

$$
x \in]-\infty,-a] \text { or } x \in[a,+\infty[.
$$

Then there exists at least one nontrivial periodic solution to (12).

Proof: Let

$$
V_{1}(x, y)=y^{2}+2 G(x), \quad(x, y) \in R^{2} .
$$

By (iii) the curves $V_{1}(x, y)=c, c \in R_{+}$, are closed, encircle $l$ and cover $R^{2} \backslash l$. Since

$$
V_{1}^{*}(x, y)=(P, Q) \cdot \underline{\operatorname{grad}} V_{1}=-2 f(x) y^{2}
$$

it is seen that in the half planes $|x| \geqslant a$ the trajectories of the system (13) pass through the curves $V_{1}(x, y)=c$ from the outside.

Suppose now for instance that

$$
[F(x)]^{2} \geqslant 8 G(x), \quad x \leqslant-a .
$$

The continuous curve $k$ given by

$y=\varphi(x)$

$$
\left.\left.=\frac{1}{2}\left(-F(x)+\sqrt{[F(x)]^{2}-8 G(x)}\right), \quad x \in\right]-\infty,-a\right]
$$

then has the property, that

$$
V(x, \varphi(x))=0, \quad x \in]-\infty,-a] .
$$

Let $P_{0}$ be the point $(-a,-b)$ for some $b>0$. Starting at $P_{0}$ we construct a spiral $S$ tending to infinity by matching together pieces of the curves $V_{1}(x, y)=c$ with linesegments, as shown in Fig. 1. The slope of the line segments is chosen in such a way that the trajectories of the system (13) intersect the spiral from the outside. Let $\Omega$ be the $p$-cell (with respect to $V$ ) containing the positive $y$ axis and let $\Gamma$ be a trajectory which contains a point of $\Omega$, say 


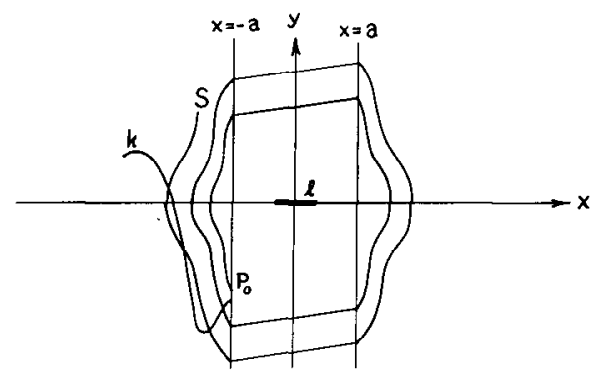

Fig. 1.

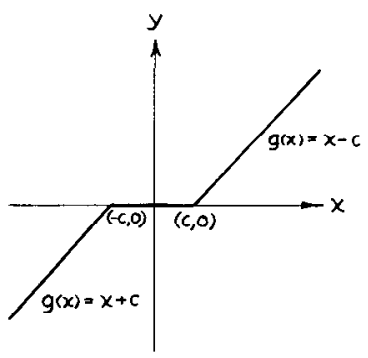

Fig. 2.

at $t=t_{0}$. Since $\Omega$ is positively invariant by (iia) $\Gamma$ cannot reach $k$ at any later time and hence $\Gamma^{+}$is bounded.

Now choose $c \in R_{+}$such that the closed curve $k_{1}$ given by $V_{1}(x, y)=c$ is contained in the strip $-\alpha_{1}<x<\alpha_{2}$. Since $V_{1}^{*}(x, y)=-2 f(x) y^{2}$ is nonnegative in this strip, the compact set $U$ bounded by $k_{1}$ is negatively invariant. Since $l$ is contained in the interior of $U$, it follows that $L\left(\Gamma^{+}\right) \cap l=\varnothing$. Hence $L\left(\Gamma^{+}\right)$is a closed trajectory.

Example: We consider a modified van der Pol equation

$$
\ddot{x}+\epsilon\left(x^{2}-1\right) \dot{x}+g(x)=0
$$

where $\epsilon$ is a positive constant and $g(x)$ is the piecewise linear function, shown on Fig. 2. It is assumed that $c \in] 0,1[$ is a constant.

This situation displays the case in which the "external force" $g(x)$ vanishes in some interval $[-c, c]$, i.e., only the "damping force" will be active in this interval.

By a straightforward calculation we get if $x>c$

$$
\begin{aligned}
\tilde{V}(x, y) & =2 G(x) f(x)-F(x) g(x) \\
& =(x-c)^{2} \epsilon\left(x^{2}-1\right)-\epsilon\left(\frac{1}{3} x^{3}-x\right)(x-c) \\
& =\epsilon(x-c)\left(\frac{2}{3} x^{3}-c x^{2}+c\right) \\
& \geqslant \epsilon c\left(1-\frac{1}{3} c^{2}\right)(x-c)>0
\end{aligned}
$$

since the polynomial $2 / 3 x^{3}-c x^{2}+c$ is increasing for $x>c$. It is seen that $2 G(x) f(x)-F(x) g(x)>0$ holds on $R \backslash[-c, c]$ since the functions $G(x) f(x)$ and $F(x) g(x)$ are both even. Hence, the conditions of Theorem 4 are all satisfied with $a_{1}=a_{2}=c$. Moreover, the leading term of

$$
[F(x)]^{2}-8 G(x)=\epsilon^{2}\left(\frac{1}{3} x^{3}-x\right)^{2}-4(x-c)^{2}, \quad x>c
$$

has positive coefficient, i.e., the condition (v) will be satisfied with some $a$ greater than 1. Condition (iv) is satisfied since $c<1$. We conclude that the conditions of Theorem 5 are all satisfied. Hence (14) has a unique nontrivial periodic solution up to translations in timc.

\section{REFERENCES}

[1] R. J. P. de Figueiredo, "Existence and uniqueness results for Liénard's equation," IEEE Trans. Circuit Theory, vol. CT-17, pp. 313-321, Aug. 1970.

[2] A. A. Andronow, A. A. Vitt, and S. E. Khaikin, Theory of Oscillators. Reading, MA: Pergamon, pp. 333-334, 1966.

[3] J. K. Hale, Ordinany Differential Equations. New York: WileyInterscience, p. 55, 1969.

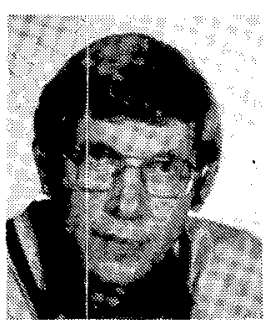

Allan Sandqvist was born in Denmark in 1942. He received the M.Sc. degree in mathematics from the University of Copenhagen in 1967.

Since 1966 he has been affiliated with the Technical University of Denmark, now as Associate Professor at the Department of Mathematics. His interests are ordinary differential equations and control theory.

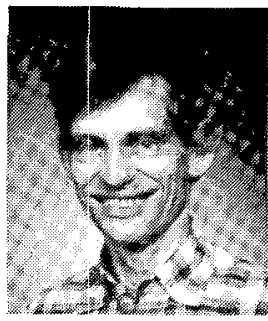

Kurt Munk Andersen was born in Denmark in 1941. He received the M.Sc. degree in mathematics from the University of Copenhagen, Denmark, in 1965.

Since the Fall of 1966 he has been affiliated at the Department of Mathematics, the Technical University of Denmark, Lyngby. From 1972 he has been an Associate Professor. His main interests are ordinary differential equations and control theory. 